\title{
When upward social mobility leads to frustration: Boudon's game-theoretic model of relative deprivation and experimental evidence
}

\author{
Kasper Otten \\ Utrecht University, Department of Sociology, Padualaan 14, 3584 CH Utrecht, the Netherlands
}

\section{Introduction}

Improvements of opportunities in a group or society can paradoxically lead to more frustration among its members. Well-known examples include the several revolutions that were preceded by social and economic improvements (Brinton, 1938; de Tocqueville, 1952 [1856]), sometimes referred to as "de Tocqueville's Paradox" (Oberschall, 1970) and the finding that American soldiers during World War II were less positive about their promotion opportunities in branches with more social mobility (Stouffer et al., 1965 [1949]). Despite such occurrences, most research so far has focused on explaining situations in which improvements of social opportunities decrease (see Stevenson \& Wolfers, 2013) or do not change (see Easterlin, 2001) levels of frustration. Research on the conditions under which improvements of opportunities lead to more frustration is scarce and inconclusive. In this paper, we examine when and how the increase of a particular opportunity in a group, namely upward social mobility (from now on referred to simply as social mobility), can paradoxically lead to more frustration among its members.

The theory of relative deprivation is commonly believed to provide the explanation for the paradox (Stouffer et al., 1965 [1949]; Runciman, 1966; Merton \& Rossi, 1968; Boudon, 1982 [1977]; Walker \& Smith, 2002). The theory suggests that frustration is determined by relative, rather than absolute, outcomes, and therefore puts focus on inter-personal comparisons. A person is relatively deprived when he/ she compares him or herself to another person, notes that he/she is disadvantaged compared to this person, and feels a negative emotion because of this (Pettigrew, 2015). An increase in social mobility within a group is hypothesized to lead to more frustration among its members when the increase benefits some more than others, causing the persons that do not benefit (as much) to feel relatively deprived.

Yet, the underlying mechanism of inter-personal comparisons hypothesized to drive the relation between more social mobility and more relative deprivation has received little theoretical and empirical scrutiny so far. An association between two macro phenomena (a group's level of social mobility and relative deprivation) that runs via microprocesses (person-level comparisons) requires explicit modelling of the micro-macro links. As of yet, there is no such empirically supported model. In this paper, we extend and experimentally examine a game- theoretic model of relative deprivation by Boudon (1982 [(1977)], pp. 105-126) that does explicitly address the underlying micro-processes and micro-macro links.

In the model, actors choose whether to make a costly investment to enter a competition for a limited number of rewards. Abstaining from investment always gives a sure payoff because the costs of investment are saved but no reward is obtained. Investing in the competition could lead one to be better or worse off than not investing: winning the reward gives a higher net payoff than not entering the competition, while failing to win a reward despite investment gives a lower net payoff. The possibility to lose in the competition exists because the number of rewards is limited; if there are more investors than rewards, some investors will not attain the reward despite their investment. Such losing investors are disadvantaged compared to some of their referent others, namely the winning investors, and therefore considered to be relatively deprived (Boudon, 1982 [1977]; Kosaka, 1986).

The model's general structure of costly competition for rewards bears resemblance to several real-life situations. For example, the rewards can be interpreted as high-status occupational positions that can be achieved through costly investments in higher education (Boudon himself also adopted such interpretations, see for example: 1982 [1977], p. 124; 1979, pp. 56-60; another example is competition for promotions within firms). Increasing one's position is done by winning a reward, and increases in the number of rewards can therefore be regarded as improvements in upward social mobility. The paradoxical relationship between social mobility and relative deprivation comes about when increasing the number of rewards (social mobility) causes a disproportionately large increase in investors, and therefore a higher proportion of losing investors (e.g., highly educated persons with a lowstatus position). After increasing social mobility so far that many actors are already investing, further improvements will lead to a disproportionally lower increase in investors, and so to lower levels of relative deprivation. The model's predicted pattern between social mobility and the macro-level of relative deprivation is thus inversely $\mathrm{U}$ shaped.

Current knowledge on the model's relationship between social mobility and relative deprivation is predominately based on analyses with behavioural assumptions of self-regarding preferences: in making their investment decision, actors are assumed to only care about their

E-mail address: k.d.otten@uu.nl. 
own outcome and not that of others. We give a new analysis of the relationship between social mobility and relative deprivation by gametheoretically introducing arguably more realistic assumptions of otherregarding preferences: actors aim to have a good outcome and to not be at a disadvantage compared to others. Additionally, we extend the competition structure of the model to analyse sources of relative deprivation in the model that have so far remained unexplored. Specifically, we change the way rewards are allocated when there are fewer investors than rewards, i.e., in situations of underinvestment. Boudon left such excess rewards unallocated. We suggest that by allocating the excess rewards among the non-investors, new sources of relative deprivation can be studied. For example in occupational structures, if there are more high-status positions than higher educated people, some of the lower educated people could receive one of the remaining high-status positions. Such actors with a high-status position despite their low education could create feelings of relative deprivation both in actors that gained a high-status position through costly higher educational investments, and in lower educated actors that were not among the lucky ones to obtain one of the excess high-status positions.

We carry out game-theoretic computer simulations on this extended model to examine how improvements in social mobility affect investments in rewards, and how this translates to group-level relative deprivation. The simulations give us precise predictions for when more social mobility leads to more relative deprivation, either through overor underinvestment. Simulations necessarily simplify the complexity of real life so as to keep the analysis tractable. This means that there is always a risk that the results would have been different if certain parts of reality that were left out of the model would have been incorporated and varied. In an attempt to address this issue, we also test the model in a laboratory experiment with human subjects. Real subjects bring with them a lot of unspecified heterogeneity, and corroboration from a lab experiment would therefore provide a valuable robustness check of the simulation results. We end the paper with a discussion of our findings and suggestions for future research, both inside and outside the lab.

\section{Theory}

\subsection{Boudon's model}

Boudon's relative deprivation model (1982 [1977], pp. 105-126) can be outlined using the following notation. There is a homogeneous group of $N$ actors. Each of these actors has to make a choice between two options. They can either invest some amount $C_{H}$ (this represents investment in for instance education) to have the possibility to reap some reward $B_{H}$ (which then represents a high-status position) $\left(B_{H}>C_{H}\right)$, or they can invest a smaller amount $C_{L}\left(C_{L}<C_{H}\right.$; a smaller investment in education) to have a smaller but sure payoff $B_{L}$ (a lowstatus position) ( $B_{L}>C_{L} ; B_{H}>B_{L}$ ). The potential gain of investing is larger than the potential gain of not investing: $B_{H}-C_{H}>B_{L}-C_{L}$. The influence of these four parameters can be summarized into a cost-tobenefit ratio of investing for the reward $\left(Q=\left(C_{H}-C_{L}\right) /\left(B_{H}-B_{L}\right)\right)$. To ease the interpretation of the model, we can assume $C_{L}=0$, such that choosing the low investment means not investing. This assumption implies no loss of generality because the actor's investment does not depend directly on $C_{L}$, but rather on the total cost-to-benefit ratio, and setting $C_{L}$ to 0 does not restrict the range of this ratio.

There is only a limited number $k$ of rewards $B_{H}$, while there are unlimited low payoffs $B_{L}$. Increasing the number of rewards $k$ is therefore generally used to represent an improvement in social mobility (Boudon, 1982 [1977]; Yamaguchi, 1998). More specifically, the number of rewards determines the chance of absolute upward movement for those who invest, without directly focusing on how this movement shifts the internal rankings between actors. In that sense, the parameter resembles absolute mobility rather than relative mobility. Similarly, because the parameter constrains the macro-level distribution of positions, it corresponds more closely to structural mobility than circulation mobility. As we will see, how changes in this upward social mobility parameter affect the actor's investment decision and subsequent relative deprivation should not be studied in isolation from the other aspects of the competition structure. All aspects of the structure, including social mobility, cost-to-benefit ratio of investment, and number of other actors, are taken into account by the actor to judge whether investment will be profitable, and should therefore be considered in the analysis of the model as well. The number of actors making the investment $C_{H}$ is labelled $n_{H}$ and the number of actors deciding not to invest (investing $C_{L}=0$ ) is labelled $n_{L}$, with $N=n_{H}+n_{L}$.

If the number of investors exactly equals the number of rewards $\left(n_{H}\right.$ $=k$ ), all investors will get a reward, and all non-investors do not. If there are more investors than rewards $\left(n_{H}>k\right)$, not every investor can get a reward. The rewards will then be randomly allocated over the investors, and the rest of the investors do not receive a reward despite having made a costly investment. The proportion of these losing investors (out of $N$ ) defines the macro-level of relative deprivation. If there are fewer investors than rewards $\left(n_{H}<k\right)$, there will be rewards left after all investors have received one, which are not allocated in the original model.

Recently, it has been argued that the operationalisation of relative deprivation as the proportion of losing investors is incomplete. It does not incorporate that non-investors without a reward have a lower net payoff than the investors with a reward, and therefore also feel relative deprivation, albeit less strongly than the investors without reward. ${ }^{1}$ To take both type types of relative deprivation into account, versions of the Gini coefficient have been suggested as operationalisation (Yitzhaki, 1979; Berger \& Diekmann, 2015), of which one is:

$R D=\frac{1}{N} \sum_{i=1}^{N} \sum_{j=1}^{N} \max \left(\left(B_{j}-C_{j}\right)-\left(B_{i}-C_{i}\right), 0\right)$

This operationalisation looks within all pairwise comparisons between actors in a group for positive differences in net payoffs (net payoffs are the benefits associated with the achieved reward minus the costs of investment). These net payoff differences are then aggregated and divided by the total number of actors in a group, to achieve an average level of relative deprivation within the group. Because this operationalisation takes each comparison, and the magnitude of the payoff difference within the comparison, into account, it includes all possible feelings of relative deprivation within a group. This is the group-level operationalisation of relative deprivation that we will use throughout the paper.

\subsection{Prior work}

Boudon's model of relative deprivation has stimulated little interest in the field of stratification research, but a number of scholars from other fields have contributed to its further analysis and development. One strand of research has developed a game-theoretic approach to the model, in which the preferences and beliefs of the actors are central. So far, it is largely assumed that actors have a preference to maximize their own payoff, and that, given their beliefs on the behaviour of the other actors, they act rationally in line with this preference. This means that actors invest in a reward if they expect that the benefits of investment exceed the costs of investment. The approach started with Raub (1984), who rigorously analysed the model under several game-theoretic solution concepts (maximin, pure strategy Nash equilibrium, mixed strategy Nash equilibrium), confirmed that the inverse U-shape relationship between social mobility and relative deprivation is common

\footnotetext{
${ }^{1}$ Boudon acknowledged that non-investors may also feel 'resigned frustration' towards investors with a reward. The choice of non-investment, he argued, does not imply that they do not desire a reward, but rather that the risks in obtaining a reward is too large. Non-investors may therefore still envy the actors that managed to achieve a reward.
} 
under these concepts, and provided precise boundary conditions for it. Kosaka (1986) introduced the assumption of common knowledge on the number of investors among actors, allowing him to analyse the model with classic mathematical techniques. He corroborated the pervasiveness of the inverse U-shape between mobility and relative deprivation, and additionally showed that relative deprivation is somewhat lower when there is actor heterogeneity in risk aversion. Yamaguchi (1998) and Reyniers (1998) continued the study of how actor heterogeneity affects the model's results, this time by studying differences between actors in the ability to win a reward and the cost-to-benefit ratio of investment. Their equilibrium analyses suggest that the paradox remains ubiquitous even when incorporating these more complex aspects of social life. Finally, Berger and Diekmann (2015) further clarified the model's game-theoretic analysis of dominant strategies and mixed strategy Nash equilibria, and conducted a first empirical test of these predictions in a laboratory experiment, which provided mixed support for the predictions.

Recently, another approach has been taken in which the model is analysed through agent-based simulations that are more flexible (at the expense of some tractability) than the prior game-theoretic approach (Manzo, 2009, 2011). In accordance with an earlier version of Boudon's model (1979), Manzo assumes that actors invest in the competition if they can expect a certain minimal gain from their investment. He elaborates on the model in several ways. First, an important distinction between relative deprivation frequency and degree is made. Relative deprivation frequency refers to the number or proportion of actors that do not obtain what they want, i.e., the original measure of relative deprivation as suggested by Boudon, while relative deprivation degree refers to the intensity of the feelings that are associated with not obtaining what one wants. It is shown that it is important to take both measures of relative deprivation into account because they may have different, and sometimes even opposing, relations with social mobility. One way to simultaneously incorporate both types of relative deprivation is by using our aforementioned Gini coefficient measure.

In addition, Manzo shows how changing the competition structure of the model can open up possibilities to examine new sources of relative deprivation. Whereas in Boudon's original model only the winner payoffs $B_{H}$ are limited in number, in Manzo's version the lower payoffs $B_{L}$ for abstaining from competition are also limited in number. If the number of actors abstaining from competition exceeds the number of low payoffs $B_{L}$, a random selection of them will receive no payoff at all. This creates a second category of relatively deprived actors: those who were among the unlucky excess of abstainers that received nothing. When applying the model to occupational structures, for example, an excess of lower educated persons for low-status positions may mean that some persons obtain no position at all. Through agent-based simulations on models with the different categories of relatively deprived actors and the two measures of deprivation (frequency and degree), it is shown that the paradoxical relationship between social mobility and relative deprivation appears in a region of the parameter space that crucially depends on the cost-to-benefit ratio of investing for the reward. Finally, it is shown that network structure also affects the model's predictions: social comparisons within local networks, as opposed to global networks, heighten relative deprivation.

\subsection{Extended model}

We first introduce new game-theoretic directions for the model related to the preferences and behaviour of actors. We then show how further changing the competition structure opens up possibilities to examine sources of relative deprivation in the model that have remained unexplored so far.

\subsubsection{Preferences}

As we have seen, a central assumption in the game-theoretic strand of research on the model is that actors want their own expected payoff to be as high as possible. This assumption of payoff maximizing behaviour helps to keep the analysis of the model tractable, but has been challenged by a large body of empirical research on other game-theoretic models. It has been suggested that behaviour is generally better modelled by assuming that actors care not only about their own payoffs, but also on the payoffs of the other actors in their group. More specifically, assuming that actors have self-centered inequity aversion considerably improves behavioural predictions in several situations (Fehr \& Schmidt, 1999). Actors with self-centered inequity aversion still like to obtain higher payoffs, but they additionally dislike it if their payoff relative to the other actors is inequitable. In the conventional self-centered inequity aversion model, actors dislike both inequitable payoffs that are in their advantage and disadvantage. We adapt this inequity aversion to Boudon's model, by assuming that actors are averse only to inequitable payoffs that are to their disadvantage. ${ }^{2}$ Actors thus want to obtain high payoffs, but also anticipate that this pursuit could lead to relative deprivation, which they dislike. This means that actors invest in a reward if they believe that the chance to obtain the reward is sufficiently high to offset the costs of investment and the possibility of relative deprivation if no reward is achieved despite investment. We formalize these preferences in a utility function that the actor tries to maximize:

$U_{i}=B_{i}-C_{i}-\frac{\theta_{i}}{N-1} \sum_{j \neq i} \max \left(\left(B_{j}-C_{j}\right)-\left(B_{i}-C_{i}\right), 0\right)$

An actor's utility is thus positively related to his/her own net payoff $\left(B_{i}-C_{i}\right)$ as in prior research on the model, but newly also decreases for each comparison with another actor that has a higher net payoff $\left(B_{j}-\right.$ $C_{j}$ ). The parameter $\theta_{i}$ expresses how sensitive an actor is to the relative deprivation in these social comparisons. We assume that this sensitivity differs per actor, and is only known to the actor him/herself. We use prior experimental data (Johansson-Stenman, Carlsson, \& Daruvala, 2002) to calibrate the distribution of this parameter among the actors. This is further described in the supplementary material S1.

\subsubsection{Behaviour}

Now that we have formalized the preferences into a utility function, we next have to specify how these preferences influence the behaviour of the actors. This is specified by selecting a game-theoretic solution concept, i.e., a behavioural rule for how actors make their decision. The most common solution concept is the Nash equilibrium, in which each actor's strategy is optimal given his/her correct belief on the strategies of all other actors. A drawback of assuming such best responses is that small shifts in parameter values (and hence expected utility of strategies) can lead to radically different strategies, which is difficult to analyse through numerical computer simulations. To make the outcomes depend more smoothly on the parameters, a quantal response equilibrium (QRE) concept can be applied (McKelvey \& Palfrey, 1995). In $\mathrm{QRE}$, a random error term $\varepsilon$ is added to the actors' utility function, creating decision noise that makes the actors 'better responders' rather than best responders. This leads to smoother response functions that are continuous and increasing in expected utility. We adopt a specific version of QRE, namely Bayesian QRE, for reasons we will now outline.

With our introduction of inequity aversion to the model, the actor's belief on how many others will invest no longer only depends on the costs of investment, benefits of a reward, and number of actors, all of which are common knowledge among the actors. The beliefs now also depend on the inequity aversion of others, for which the actor does not have complete information. Our modifications of the actor's preferences

\footnotetext{
${ }^{2}$ We do not assume that actors are averse to unequal payoffs that are in their advantage, because actors are in competition with each other and in that sense should not experience disutility from coming out as winner. Fehr and Schmidt (1999, p. 5) acknowledge this possibility, but do not consider it because it has no impact on equilibrium behaviour in the games they examine.
} 
therefore yield a game with heterogeneity and incomplete information with respect to the inequity aversion parameter $\theta_{i}$. The standard solution concept in this case is the Bayesian-Nash concept (Harsanyi, 1968). This is why we integrate the Bayesian-Nash approach with the aforementioned QRE concept (together referred to as BQRE). In BQRE, actors can be categorised in different types according to their personal $\theta_{i}$ but their type is private information. Given the strategic nature of the game, the actor's strategy depends on his/her own type and on his/her belief over the distribution of types (plus the game parameters and decision noise). We assume that, although each actor's sensitivity to inequity is private information, the overall distribution of inequity aversion is known to the actors. Given the information on the game settings (the costs of investment, benefit of a reward, number of actors, noise, etc.) and the distribution of inequity aversion across actors, each actor will correctly predict how many others will on average invest (the average probability of investment). Based on this information, each actor will calculate the chance of a reward under investment and non-investment, and the expected level of disadvantageous inequity under both situations. They then set their investment strategy such that their own expected utility is maximized. When every actor acts according to these rules, nobody has an incentive to deviate from their chosen strategy, and an equilibrium is achieved. More details on these analyses are provided in supplementary material S2.

\subsubsection{Competition structure}

Previously we described how Manzo's change in the competition structure of the model gave insight into a new category of relatively deprived actors, i.e., those who were among the excess number of abstainers that obtained no payoff (low or high) at all. We now aim to show how a somewhat different change in the competition structure gives insight into a yet unexplored category of relatively deprived actors. The modified competition structure is as follows.

As with Manzo's modification, the low payoffs $B_{L}$ are no longer unlimited in number. Instead, there is a fixed number of low payoffs and a fixed number of rewards (high payoffs), which together equal the population size. If there are more investors than rewards $\left(n_{H}>k\right)$, there are insufficient rewards and excessive low payoffs. A random selection of the investors $\left(n_{H}-k\right)$ will therefore be allocated to the excess low payoffs. Newly, if there are fewer investors than rewards $\left(n_{H}<k\right)$, there are insufficient low payoffs and excess rewards. The rewards are then first allocated to the investors, and those that remain $\left(k-n_{H}\right)$ are randomly allocated among the non-investors. This may happen in occupational structures, for example, when there are not enough highly educated persons to fill all the high-status positions and some of the lower educated persons obtain the remaining high-status positions. Our extension has two main implications for the model.

First, the extension introduces two new sources of relative deprivation in situations where there are fewer investors than rewards (underinvestment). Non-investors with a reward (e.g., actors with a highstatus position despite low educational investments) may create feelings of relative deprivation both in investors with a reward (e.g., actors with the same high-status position but achieved through higher educational investments) and non-investors without a reward (e.g., actors with low educational investment and low-status position). The first group may feel relatively deprived because they obtained the reward by costly investment rather than without, and the second group may feel relatively deprived because they were not among the lucky ones who were selected to obtain the reward without investment. Table 1 presents for all possible pairwise comparisons between actors the extent of relative deprivation generated, in terms of net payoff differences between outcomes.

Second, the allocation of excess rewards in situations of underinvestment adds an interesting new behavioural dynamic. In the original model without allocation of excess rewards, no investment is always the safe choice because it gives you a sure outcome (low payoff at no cost), and investment is always the risky option because it could
Table 1

Individual Level of Relative Deprivation for all Possible Comparisons Between Ego and Referent.

\begin{tabular}{llllll}
\multicolumn{5}{c}{ Referent } \\
\hline Ego & $B_{L} C_{L}$ & 0 & $B_{L} C_{H}$ & $B_{H} C_{H}$ & $B_{H} C_{L}$ \\
& $B_{L} C_{H}$ & $C_{H}-C_{L}$ & 0 & $\left(B_{H}-C_{H}\right)-\left(B_{L}-C_{L}\right)$ & $B_{H}-B_{L}$ \\
& $B_{H} C_{H}$ & 0 & 0 & 0 & $*$ \\
& $B_{H} C_{L}$ & 0 & $*$ & 0 & $C_{H}-C_{L}$ \\
& & & 0 & 0 \\
\hline
\end{tabular}

Persons can be categorised in four types according to the combination of their investment choice (low $C_{L}$ or high $C_{H}$ ) and presence of reward (low payoff $B_{L}$ or reward $B_{H}$ ). Persons of type $B_{L} C_{L}$, for example, made no investment (remember we use $C_{L}=0$ ) and obtained the low payoff $B_{L}$. The combination of type $B_{L} C_{H}$ (investor without a reward) and $B_{H} C_{L}$ (non-investor with a reward) has an asterisk because these types cannot co-exist within a group: type $B_{L} C_{H}$ only exists in situations of overinvestment, type $B_{H} C_{L}$ only in situations of underinvestment.

leave you either better off (reward by investing) or worse off (low payoff despite investing) than not investing. In the extended model, the choice of no investment can also be made as a risky decision. The reason is that some actors may strategically try to obtain a reward without investment. This is risky because not investing decreases your chance of obtaining the reward (remember that the rewards first go to investors), but could potentially save you the cost of investment. We discuss the behavioural dynamics further in Section 2.5 on the simulation results.

It is worth mentioning that, when the model is applied to occupational status attainment, there are also theoretical motivations for allocating the excess rewards (high-status positions) among non-investors (those who do not invest in higher education). In the original model, specific levels of education are always seen as a prerequisite for highstatus positions regardless of the competition that there may or may not be for such positions. The alternative perspective is that specific levels of education should not be seen as prerequisites for specific occupations, but rather as an advantage over others that do not have the same level of education. Job competition theory (Thurow, 1976), for example, suggests that the allocation of jobs to workers happens through a competition process. Very simply put, there is a queue of potential workers in order of important background characteristics (such as educational attainment), and a queue of potential positions in order of the attached rewards (such as status and wage). Employers give the top positions to those with the highest qualifications, and the positions with lower-status to those with worse qualifications. This means that in case of underinvestment, the best jobs are still assigned to those with the best relative qualifications, even if absolute qualifications are low. Screening and signalling theory (Arrow, 1973; Stiglitz, 1975) is also compatible with this perspective. Education is then seen as a signal of quality of a person, because persons with higher ability find it more easy to achieve a high level of education. As with job competition theory, persons with low absolute levels of education can still get selected for high-status positions, as long as their level of education relative to others is not low. What is more, in his famous model of education, opportunity, and social inequality (1974), Boudon did assign excess high-status positions to persons that did not make the matching educational investment. We will now examine the implications of incorporating this rule in Boudon's model of relative deprivation through computer simulations.

\subsection{Simulation design}

We are interested in how increasing social mobility in the model (the proportion of rewards) affects two main outcomes of the model: the group-level probability of investment $p_{C H}$ and relative deprivation $R D$. $R D$ is the group outcome of interest, and $p_{C H}$ the actor behaviour 
causing it. To examine these relationships, we numerically calculate the equilibrium of the model under multiple parameter values. In each instance, we set the model's parameters to specific values of interest, calculate the equilibrium, and thereby derive the expected $p_{C H}$ and $R D$. Once we have done this for all parameter values of interest, we apply non-parametric regression to see how differences in parameter values relate to differences in $p_{C H}$ and $R D$.

We vary three parameters of the game in a full-factorial design: the proportion of rewards among the total number of actors $(\kappa=k / N$; this represents the social mobility) between .1 and .9, the cost-to-benefit ratio of investing for the reward $\left(Q=\left(C_{H}-C_{L}\right) /\left(B_{H}-B_{L}\right)\right)$ between 1/ 12 and 11/12, and the number of actors $N$ between 4 and 1000 . We believe this broad range of values captures most of the model dynamics. The simulation results will apply to this range only, and cannot be extrapolated to situations outside of this range. Note that realised behaviour may vary between simulation runs even when using the same set of parameter values due to the stochasticity of equilibrium behaviour. The expected equilibrium behaviour, however, is always the same in each run with the same parameter values, and because we analyse this expected behaviour we only examine each combination of parameter values once. More details on the simulation design can be found in supplementary material S3.

We present the results of the non-parametric regressions in Fig. 1, through plots that illustrate the average marginal effects of social mobility $\kappa$ on the outcome of interest $\left(p_{C H}\right.$ or $\left.R D\right)$. The effects are shown for four values of the cost-to-benefit ratio of investment $Q$ : a low (4/12), medium (6/12), medium-high (8/12) and high (10/12) value that together give a representative view of how the relationship between social mobility and the outcome of interest $\left(p_{C H}\right.$ or $R D$ ) depends on the cost-to-benefit ratio of investment. Colored $3 \mathrm{~d}$ plots that show the results of all our estimated parameter combinations are given in supplementary material S3. We only present plots for $N=1000$, as variation in $N$ does not substantively affect the results (see supplementary material S4).

\subsection{Simulation results}

\subsubsection{Low to medium social mobility}

Fig. 1a shows that as long as social mobility is at low or medium levels $\left(p_{C H} \lesssim .5\right.$ ), there will be overinvestment (all the black investment lines are then above the grey line that indicates investments proportionate to rewards). This means that rewards will only be allocated to investors, and that some of the investors will receive no reward despite

(a) Probability of investment $\left(p_{C H}\right)$

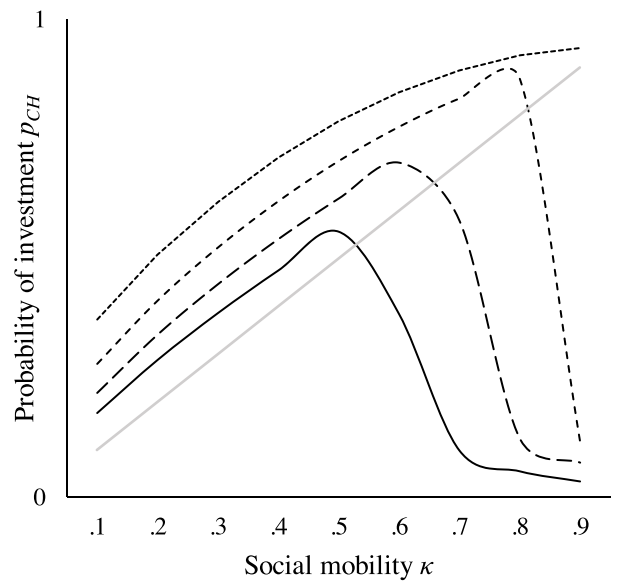

their investment. The figure further shows that if social mobility increases from a low $\left(p_{C H} \approx .1\right)$ to medium level $\left(p_{C H} \approx .5\right)$, the probability of investment always increases. This generally shifts the actors from a situation of equality (majority of actors with no reward) to a situation of inequality (about half of actors without reward and half with reward). Fig. 1b shows that this increasing inequality of rewards when moving from a low to medium level of social mobility leads to an increase in relative deprivation if the cost of investment is not high (high-cost line is with $Q=10 / 12$ ). Then, the investors with a reward are substantially better off than non-investors without a reward. If the cost of investment is high, the net differences in payoffs between investors with a reward and non-investors without a reward are small. Relative deprivation then remains largely stable when moving from low to medium levels of social mobility, despite the increasing inequality of rewards.

\subsubsection{Medium to high social mobility}

Depending on the cost of investment, further increasing social mobility from a medium $\left(p_{C H} \approx .5\right)$ to a high level $\left(p_{C H} \approx .9\right)$ either leads to a higher or lower probability of investment. If the cost of investment is low (line with $Q=4 / 12$ ), the probability of investment further increases. This shifts the actors from the situation of inequality back to a situation of equality again, where the majority of the actors obtain a reward. Accordingly, the net payoff differences between the actors decrease. The total path of relative deprivation over increasing social mobility is thus inversely U-shaped if the cost of investment is low. If the cost of investment is not low, the probability of investment will drop somewhere in the increase of social mobility from a medium to high level. This happens because actors will strategically start attempting to obtain a reward without investment when the rewards are prevalent and investment is expensive. The overall path of the probability of investment over social mobility is thus inversely U-shaped if the investment costs are not low. This has two subsequent effects on relative deprivation.

First, the drop in the probability of investment causes it to match the proportion of rewards (i.e., $p_{C H}=\kappa$; in Fig. $1 \mathrm{~b}$ where an investment line intersects with the grey line). This causes a short steep decrease in relative deprivation, as it swiftly erases the actors that were worst off: investors without a reward. Second, further increasing social mobility causes the number of investing actors to fall below the number of rewards, creating a selection of actors that will now obtain a reward without investing. These actors receive a better outcome than both the non-investors without reward and the investors with a reward. This

(b) Relative deprivation $(R D)$

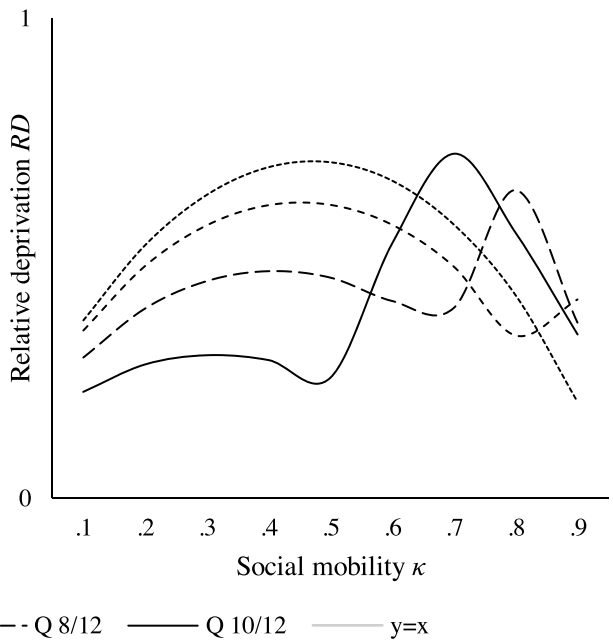

Fig. 1. (a-b) Outcome $\left(p_{C H}\right.$ or $R D$ ) as a Function of Social Mobility (the Proportion of Rewards, $\kappa$ ) and Cost-to-benefit Ratio of Investment $(Q)$, for $N=1000$. 
Table 2

Hypotheses and Point Predictions for the Outcomes $\left(p_{C H}\right.$ and $\left.R D\right)$.

\begin{tabular}{|c|c|c|c|c|c|c|c|c|}
\hline \multirow[t]{2}{*}{ Outcome } & \multirow[t]{2}{*}{$Q=$ Cost-to-benefit ratio } & \multicolumn{3}{|c|}{ Point predictions } & \multicolumn{4}{|c|}{ Directional predictions } \\
\hline & & $\begin{array}{l}\kappa \\
=1 / 6\end{array}$ & $\begin{array}{l}\kappa \\
=3 / 6\end{array}$ & $\begin{array}{l}\kappa \\
=5 / 6\end{array}$ & $\begin{array}{l}\kappa \\
1 / 6 \rightarrow 3 / 6\end{array}$ & Hypothesis nr. & $\begin{array}{l}\kappa \\
3 / 6 \rightarrow 5 / 6\end{array}$ & Hypothesis nr. \\
\hline$p_{C H}$ & low & .52 & .83 & .85 & + & H1a & 0 & H1b \\
\hline$p_{C H}$ & high & .22 & .44 & .05 & + & $\mathrm{H} 1 \mathrm{c}$ & - & H1d \\
\hline$R D$ & low & 13.66 & 21.40 & 11.73 & + & $\mathrm{H} 2 \mathrm{a}$ & - & $\mathrm{H} 2 \mathrm{~b}$ \\
\hline$R D$ & high & 6.20 & 9.03 & 10.53 & 0 & $\mathrm{H} 2 \mathrm{c}$ & 0 & $\mathrm{H} 2 \mathrm{~d}$ \\
\hline
\end{tabular}

$p_{C H}=$ probability of investment. $R D=$ relative deprivation. $\kappa=$ proportion of rewards, i.e., social mobility.

leads to larger interpersonal differences and therefore an increase in relative deprivation again. This increase stops when the probability of investment has dropped so far that the distribution of outcomes among actors becomes more equal again: the majority of actors now get a reward without investment. When the proportion of rewards approaches 1 , almost all actors receive a reward for free, bringing relative deprivation again to a low point. Altogether, if the cost of investment is not low, we thus observe two consecutive inverse U-shapes for the path of relative deprivation under increasing social mobility. The first over increasing and decreasing interpersonal differences resulting from increases in investments, the second over increasing and decreasing interpersonal differences resulting from decreases in investments.

\subsection{Simulation: hypotheses}

Simulations reduce the complexity of real human behaviour to keep the analysis understandable. There is unfortunately no guarantee that human subjects will behave in the same way as the computerized actors that we implemented. A valuable robustness check of the model is therefore to test it with real subjects in a laboratory experiment. A laboratory experiment allows us to tightly control the competition structure in which subjects behave, while letting the participants free in how they choose to react to this structure. It is not possible to test all of the simulation paths in a single study; the constellations of conditions bringing them about are too numerous. We therefore test a subset of them in the laboratory experiment. In general, the predicted paths that will be tested are: when the cost-to-benefit ratio of investing is low, increasing social mobility brings about increases in investments and an inversely U-shaped path of relative deprivation; when the cost-to-benefit ratio of investing is high, increasing social mobility brings about an inversely U-shaped path of investments, and more-or-less stable levels of relative deprivation. We now turn to the parameter values and hypotheses used to test these paths in the experiment.

We design the laboratory experiment such that the conditions under which participants act, resemble the conditions of the model. We then impose specific parameter values in the experiment and examine whether the predicted probability of investment and level of relative deprivation match with what we observe in the experiment. The parameter values used in the experiment are presented in Table 2, and further discussed in the data and methods section. We vary the cost-tobenefit ratio of investing $Q$ between a low (1/4) and high (5/6) value and the proportion of rewards $\kappa$, i.e., social mobility, between a low (1/ $6)$, medium (3/6), and high value (5/6), leading to a total of six conditions. The predicted relation between social mobility on the one hand, and the probability of investment and relative deprivation on the other hand, is presented in Fig. 2.

Based on Fig. 2, we propose eight directional hypotheses. We present the hypotheses through Table 2, for which an example reading now follows. Hypothesis H1a concerns the changes in the probability of investment when social mobility moves from the low $(\kappa=1 / 6)$ to medium $(\kappa=3 / 6)$ level. The prediction ' + ' indicates that the probability of investment $p_{C H}$ is expected to increase. A prediction '-' would indicate an expected decrease, and ' 0 ' would indicate that we expect the outcome to stay more-or-less the same. We consider an outcome to stay more-or-less the same when the change between conditions is less than $7.5 \%$ of the maximum possible change (for $p_{C H}=.075$, for $R D=6$ ). ${ }^{3}$

Next to these directional hypotheses, we will also test whether the theory-based point predictions of the probability of investment and relative deprivation equal the observed numerical outcomes within each of the six conditions, i.e. human subjects behave exactly as in our models. The point predictions can be found in Fig. 2 and Table 2 .

It may be noted that the imposed change in social mobility is somewhat extreme if the model is applied to society at large. A shift in society from a situation in which only $1 / 6$ of the population is able to move up the social ladder to a situation in which $3 / 6$, or $5 / 6$, of the population is able to move up the social ladder is not very likely in the short term. Yet, if the model is applied to other contexts, such shifts are not exceptional. The level of promotions in organisations, for example, can vary considerably over time as a result of economic and business cycles (e.g., Rosenbaum, 1979; Solon, Whatley, \& Stevens, 1997). What is more, the model also predicts changes in investments and relative deprivation under small changes in social mobility, e.g., from .45 to .50. However, the effect sizes of such changes are smaller, which makes it more difficult to find them in laboratory experiments where it would put extensive requirements on the number of participants. Additional tests of the model with field data under a broader range of values would therefore be interesting. We come back to this issue in the discussion section.

\section{The experiment: data and methods}

\subsection{Subjects}

We conducted a computer-aided laboratory experiment in the Experimental Laboratory for Sociology and Economics (ELSE) at Utrecht University. The experiment was programmed and conducted with z-Tree software (Fischbacher, 2007). We recruited 144 subjects amongst students at Utrecht University using the internet recruitment system ORSEE (Greiner, 2015). The subjects played a game under several conditions. A session lasted on average 40 minutes, and subjects received on average 9 euros. The amount they earned depended on their decisions and randomness. Virtually all subjects were students at Utrecht University, 52 were Dutch and 92 from various other countries. Subjects were on average 24 years old, and 39 of them were male.

\subsection{Procedure}

Subjects participated in one of 7 sessions conducted in the winter of $2017 / 2018$. They were randomly placed in an individual cubicle, so

\footnotetext{
${ }^{3}$ Inevitably, choosing a value of negligible difference carries with it some arbitrariness. However, it is less arbitrary than only considering an estimated difference of exactly 0 as negligible. Choosing somewhat different values than $7.5 \%$ (e.g., 5 or 10 ) does not substantively affect our conclusions.
} 
(a) Probability of investment $\left(p_{C H}\right)$

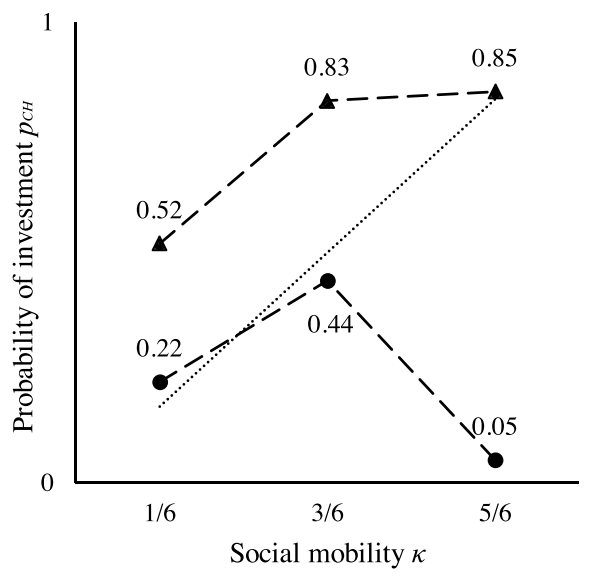

$-\mathbf{- l o w} \mathrm{Q} \quad-\bullet-$ high $\mathrm{Q} \quad \cdots \cdots \cdots \cdots \cdots \cdot \mathrm{y}=\mathrm{x}$ (b) Relative deprivation $(R D)$

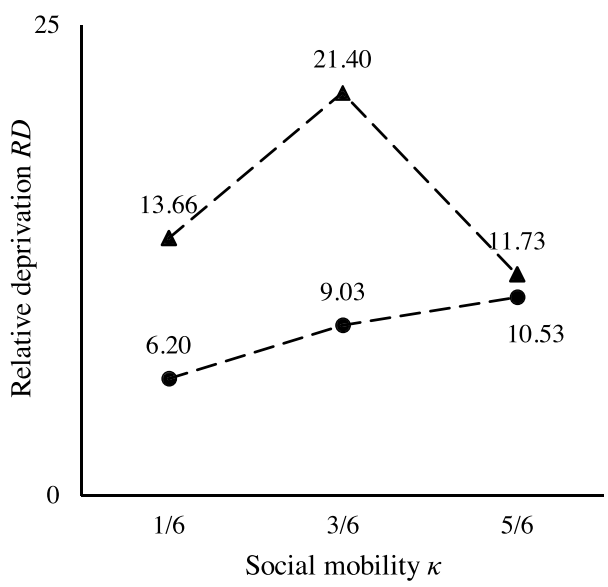

Fig. 2. (a-b) Predicted Outcome $\left(p_{C H}\right.$ or $\left.R D\right)$ Under Imposed Values of Social Mobility $(\kappa)$ and Cost-to-benefit Ratio of Investment $(Q)$.

they could not see, or communicate with, each other. Through written instructions, they were informed that they would play a competition game in groups of six. This group size is chosen such that it is big enough for statements about relative deprivation within groups, but also small enough to gather enough groups. Subjects were informed that there were six rounds of the competition game in total, and that the groups would be randomly reassigned every round. The game is as follows. In every round, there are a number of rewards to be allocated among the group. Subjects receive a budget that they can invest to participate in a competition for these rewards, or they can keep it and not participate in the competition. Investing the budget represents the cost of (high) investment of our model $C_{H}$, while keeping the budget represents no (low) investment $C_{L}(=0)$.

The rewards are first allocated to the subjects who participated in the competition. If in a round the number of investors exactly equals the number of rewards, all investors will get a reward minus their invested budget (type $B_{H} C_{H}$ ), and all subjects that did not participate in the competition (the non-investors) do not get a reward but keep their budget (type $B_{L} C_{L}$ ). If there are more investors than rewards, not every investor can get a reward. The rewards will then be randomly allocated over the investors. The rest of the investors will not receive a reward, despite having spent their budget to participate in the competition (type $B_{L} C_{H}$ ). If there are fewer investors than rewards, there will be rewards left after all investors have received a reward. A random selection of non-investors will be chosen to get one of the excess rewards, despite having kept their budget (type $B_{H} C_{L}$ ). After all subjects made their decision in a round, the rewards were allocated and the subjects informed on the outcomes for themselves and the decisions and outcomes for their group members.

After reading the instructions, subjects were given 5 questions to test their understanding of the game. Upon completion, they were shown which questions they had answered correctly. 128 subjects had all answers correct, 12 subjects had 4 answers correct, and 4 subjects had 3 or 2 answers correct. One trial round of the game was played after these questions, to further get acquainted with the game. At the end of the experiment, subjects were asked to report their understanding of the experiment. 138 reported a good understanding, 6 reported partial understanding, and nobody reported no understanding. These figures give us confidence that the experiment was adequately understood. Throughout the experiment, subjects had the opportunity to ask questions to the lab official. Only two subjects did so. The instructions and test questions with correct answers, together with the data, are available under open access in (Otten, 2018).

\subsection{Methods}

We varied the number of rewards and the cost-to-benefit ratio of investing across the six rounds. The number of rewards varied across three values: 1,3 , and 5, which represent low, medium, and high levels of social mobility. The cost-to-benefit ratio of investing varied between a low $(1 / 4)$ and high value $(5 / 6)$. For the low cost-to-benefit ratio, we set the value of the reward to 80 points and the budget to 20 , for the high cost-to-benefit ratio we set the value of the reward to 60 points and the budget to 50 . The exchange rate was 35 points for 1 euro. To ease readability, we will refer to the low and high cost-to-benefit ratios of investment as low and high investment costs. The combination of the three possible numbers of rewards and two possible investment costs leads to six possible conditions, and each subject played in each condition once. The conditions are chosen so that actors are expected to invest in such a way that situations with insufficient, sufficient, and excessive rewards are all predicted to occur. The parameter values for each condition are shown in Table 3.

Every subject played three consecutive rounds with high investment costs and three consecutive rounds with low investment costs, but it was randomly varied across sessions whether the subjects began with low or high costs. The order of the number of rewards was also randomly varied across sessions. We did not show subjects the total points they had earned over prior rounds, in an attempt to diminish prior experience affecting decisions in subsequent rounds. We find little evidence for such experience effects; the (absolute) within-subject Pearson correlations between one's outcomes in the prior rounds and the investment decision in the current round are never above .22 (see Table A1 in the Appendix).

\subsection{Measurements}

\subsubsection{Investment choice}

This binary variable indicates whether the subject invested the budget (1) or not (0) within a round.

\subsubsection{Relative deprivation}

We obtain a subject's level of relative deprivation within a round by summing all positive net differences in payoffs between the other group members and the subject in that round, and then dividing them by the 
Table 3

The Experiment: Parameters and Observations.

\begin{tabular}{|c|c|c|c|c|c|c|}
\hline & \multicolumn{6}{|c|}{ Condition } \\
\hline & 1 & 2 & 3 & 4 & 5 & 6 \\
\hline Reward value & 80 & 80 & 80 & 60 & 60 & 60 \\
\hline Budget value & 20 & 20 & 20 & 50 & 50 & 50 \\
\hline Outcome $B_{H} C_{H}$ & 80 & 80 & 80 & 60 & 60 & 60 \\
\hline Outcome $B_{H} C_{L}$ & 100 & 100 & 100 & 110 & 110 & 110 \\
\hline Outcome $B_{L} C_{H}$ & 0 & 0 & 0 & 0 & 0 & 0 \\
\hline Outcome $B_{L} C_{L}$ & 20 & 20 & 20 & 50 & 50 & 50 \\
\hline Cost-to-benefit ratio $\left(C_{H}-C_{L} / B_{H}-B_{L}\right)$ & $1 / 4$ & $1 / 4$ & $1 / 4$ & $5 / 6$ & $5 / 6$ & $5 / 6$ \\
\hline Number of rewards $k$ & 1 & 3 & 5 & 1 & 3 & 5 \\
\hline Group size $N$ & 6 & 6 & 6 & 6 & 6 & 6 \\
\hline Proportion of rewards $(k / N)$ & $1 / 6$ & $3 / 6$ & $5 / 6$ & $1 / 6$ & $3 / 6$ & $5 / 6$ \\
\hline Nr. of observations & 144 & 144 & 144 & 144 & 144 & 144 \\
\hline Nr. of groups & 24 & 24 & 24 & 24 & 24 & 24 \\
\hline Total nr. of observations & & & & 54 & & \\
\hline Total nr. of subjects & & & & 44 & & \\
\hline Total nr. of groups & & & & 44 & & \\
\hline
\end{tabular}

number of other group members:

$R D_{i}=\frac{1}{N-1} \sum_{j=1}^{N} \max \left(\left(B_{j}-C_{j}\right)-\left(B_{i}-C_{i}\right), 0\right)$

An example calculation follows. Take a (male) subject who did not invest his budget of 20 points and did not get a reward $\left(B_{i}-C_{i}=20\right)$. He has 1 group member that also did not invest and did not get a reward $\left(B_{j}-C_{j}=20\right), 3$ group members that invested and got a reward of 80 points $\left(B_{j}-C_{j}=80\right)$, and 1 group member that invested but did not get a reward $\left(B_{j}-C_{j}=0\right)$. Only the 3 group members that invested and got a reward have a higher net payoff than ego, and therefore lead to relative deprivation. The net difference between him and each of these three group members is 60 , leading to a combined difference of 180 . This score is divided by the number of other group members (5), leading to a relative deprivation score of $R D_{i}=36$. In supplementary material S5, we test and confirm that relative deprivation as we measure it is associated with feelings of dissatisfaction, anger, sadness, and injustice, which are all common negative feelings induced by relative deprivation (Smith, Pettigrew, Pippin, \& Bialosiewicz, 2012).

Table 4 presents the descriptive statistics for the investment and relative deprivation variables. The frequencies of the type of subjects are also included.

\subsection{Analyses}

Our simulations focused on the mean behaviour and level of relative deprivation over a population of actors, rather than on how behaviour and relative deprivation vary between actors. In line with this focus, we analyse the experiments with population-averaged (PA) models (Zeger, Liang, \& Albert, 1988). Rather than using the observations nested within subjects to receive subject-specific (conditional) estimates,

Table 4

Descriptive Statistics for Experiment, Subject id: 1..144, Round 1..6, Condition: 1..6.

\begin{tabular}{llllll}
\hline & $\mathrm{N}$ & Mean & $\mathrm{SD}$ & Min & Max \\
\hline Investment choice & 864 & .58 & - & 0 & 1 \\
Relative deprivation & 864 & 13.19 & 18.32 & 0 & 80 \\
Type $B_{L} C_{L}$ & 864 & .34 & - & 0 & 1 \\
Type $B_{L} C_{H}$ & 864 & .16 & - & 0 & 1 \\
Type $B_{H} C_{H}$ & 864 & .42 & - & 0 & 1 \\
Type $B_{H} C_{L}$ & 864 & .08 & - & 0 & 1
\end{tabular}

population-averaged models provide marginal outcomes averaged over the population of subjects, while also taking account of repeated measures within subjects. Consequently, as in the simulations, the estimates have an interpretation in terms of the response averaged over the population (Hardin \& Hilbe, 2003). This means that, for example, the estimated level of relative deprivation within a certain condition is not interpreted as the observed relative deprivation of a subject with specific attributes, but rather of the average subject. The relative deprivation of an average subject within a group is equal to the average relative deprivation across all subjects of that group, which is why we can interpret the estimates of relative deprivation on the (macro) group-level.

To test our hypotheses on the probability of investment (H1a-H1d), we use a population-averaged logistic regression with a factor representing the six conditions as the independent variables. To test our hypotheses on relative deprivation $(\mathrm{H} 2 \mathrm{a}-\mathrm{H} 2 \mathrm{~d})$, we use a populationaveraged linear regression with the same independent variables. In both regression models, we control for session effects. All hypotheses that predict differences between conditions are evaluated by null-hypothesis testing of pairwise differences between the predictive margins of the conditions. All hypotheses of equivalence between conditions, or between predicted and observed points (point predictions), are evaluated by equivalence testing with the two one-sided tests method (see Walker \& Nowacki, 2011). In this method, one specifies a theoretical difference that is considered to be negligible (we use $7.5 \%$ of the maximum outcome, in line with hypotheses construction in Section 2.5), and then adds and subtracts this threshold to and from the point estimate, to get a range of equivalence. If the $90 \%$ confidence interval of the observed point falls within this range of equivalence, the predicted and observed point are said to be significantly equivalent.

\section{Results}

The estimates for the hypotheses tests are provided in the appendix (Tables A2-A4 for the directional hypotheses, Table A5 for the point hypotheses). Tests of significance will be based on these estimates. We illustrate the results through Fig. 3, that depicts both the predicted (dashed lines) and observed (solid lines) relations between the number of rewards $k$ ( $x$-axis) and the outcome of interest ( $y$-axis), under low (triangle points) and high (circle points) investment costs. ${ }^{4}$

The figure suggests that there is a large role of investment costs in determining whether there is over- or underinvestment (indicated by whether the investment lines are above or below the dotted line of proportionate investments). With low investment costs, there is always overinvestment, with high investment costs there is almost always underinvestment. Relative deprivation occurs in both situations. Overall, the predictions seem to match the observations rather closely. For a thorough evaluation, however, we need to analyse the data using statistical tests.

\subsection{Directional hypotheses}

We start with analysing the probability of investment (Fig. 3a). Under low investment costs, we predicted the probability of investment to increase when moving from the low $(k=1)$ to medium $(k=3)$ level of social mobility (H1a), and then to remain stable when moving from the medium to high $(k=5)$ level $(\mathrm{H} 1 \mathrm{~b})$. We find a significant increase in the probability of investment from .49 to .88 when social mobility moves from the low to medium level, supporting hypothesis H1a. The increase in the probability of investment from .88 to .93 when moving from the medium to high level of social mobility is not significant, but

\footnotetext{
${ }^{4}$ The observed points are taken from the predictive margins in Table A2. The difference with the descriptive results is negligible ( $\leq .03$ for $p_{C H}$ and $\leq .15$ for $R D)$.
} 
(a) Probability of investment

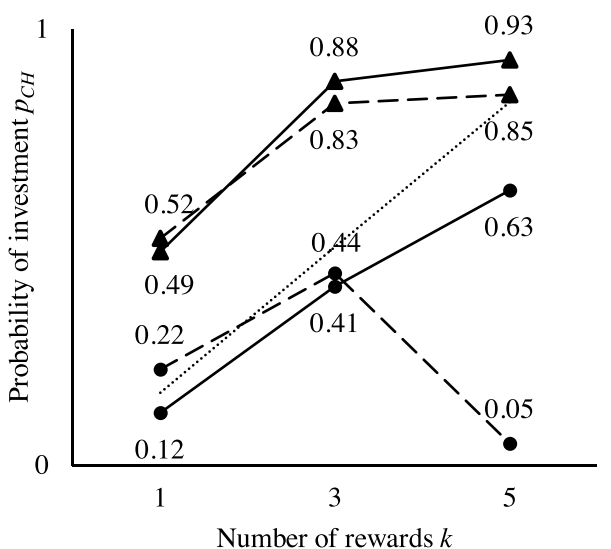

Number of rewards $k$ (b) Relative deprivation

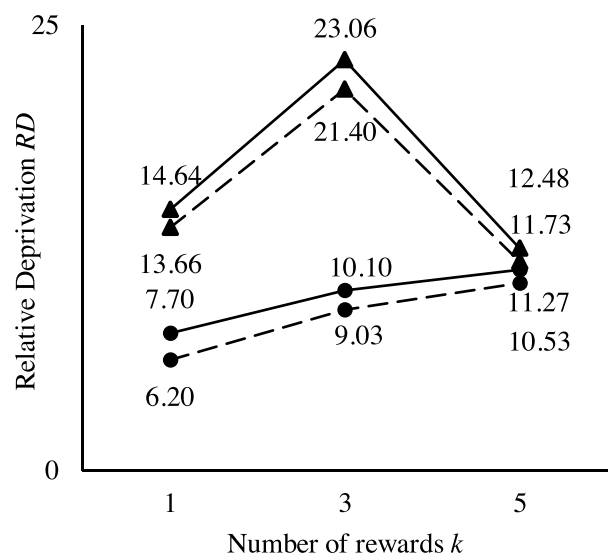

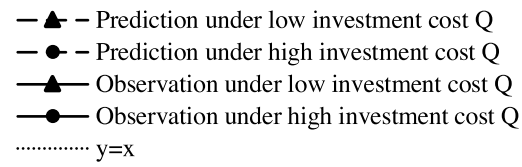

Fig. 3. (a-b) Predicted and Observed Outcomes Experiment.

neither are these probabilities significantly equivalent. We thus do not find convincing support for hypothesis H1b.

Under high investment costs, we predicted the probability of investment to increase when moving from the low to medium level of social mobility (H1c), and then to decrease when moving from the medium to the high level (H1d). We find a significant increase in the probability of investment from .12 to .41 when social mobility moves from the low to medium level, supporting hypothesis H1c. We find a further significant increase to .63 when moving to the high level, rejecting hypothesis H1d. To summarize the investment results, we find that we can correctly predict the change in investments when social mobility improves from the low to medium level (H1a and H1c), but mostly not when improving social mobility from the medium to high level (H1b and H1d).

We now turn to relative deprivation (Fig. 3b). Under low investment costs, we predicted relative deprivation to increase when moving from the low to medium level of social mobility (H2a), and then to decrease when moving from the medium to the high level (H2b). We find that relative deprivation increases significantly from 14.64 to 23.06 when social mobility moves from the low to medium level, and then decreases significantly to 12.48 when moving to the high level. This supports hypotheses H2a and H2b. Note that hypothesis H2b is supported even though the associated hypothesis on the underlying investment behaviour (H1b) was not strongly supported; we come back to such mismatches in Section 4.3. Under high investment costs, we predicted relative deprivation to remain stable both when social mobility increases from a low to medium (H2c) and medium to high level (H2d). We find the relative deprivation score of 7.70 under the low level of social mobility to be significantly equivalent to the relative deprivation score of 10.10 under the medium level of social mobility, supporting hypothesis H3c. This last relative deprivation score is also significantly equivalent to the relative deprivation score of 11.27 under the high level of social mobility, supporting Hypothesis H2d. Note that hypothesis $\mathrm{H} 2 \mathrm{~d}$ is supported despite rejection of the hypothesis underlying actor behaviour (H1d). To summarize, we find relative deprivation under low investment costs to increases when social mobility moves from the low to medium level, and then to drop when moving to the high level, leading to an inversely U-shaped path. Under high investment costs, improvements of social mobility leave relative deprivation mostly unaltered.

\subsection{Point predictions}

We find little support for equivalence between the predicted and observed probabilities of investment within conditions. This means that, notwithstanding the partial support for the directional predictions, the point predictions for the probability of investment are unsupported - the game-theoretic model does not yield accurate numerical predictions of behaviour. We do find considerable support for equivalence between the predicted and observed relative deprivation scores. All predicted values are significantly equivalent to the observed values. The point predictions for relative deprivation are thus supported. Choosing a different threshold for equivalence than $7.5 \%$ of the maximum outcome, for example 5 or $10 \%$, does not substantively affect support of the point predictions.

\subsection{Mismatches empirical support investment behaviour and relative deprivation}

It is apparent that the predictions on relative deprivation receive more support than the predictions on investment behaviour. This mismatch is an interesting finding, because it suggests that our predicted patterns of relative deprivation are more general than the underlying actor behaviour we hypothesized to cause them. To illuminate how such differences can come about we discuss the major case of mismatch here, which happens in the combination of the high investment cost and the high level of social mobility $(k=5)$. Here, we predicted virtually all subjects to abstain from investment to obtain the rewards for free $\left(p_{C H}\right.$ $=.05$ ). If that were the case, 5 out of 6 subjects within each group would have obtained a reward for free and feel no relative deprivation, while 1 subject within each group would have obtained no reward and feel a strong sense of relative deprivation as the result of comparing to five subjects with free rewards. In actuality, about 4 out of 6 subjects invest $\left(p_{C H}=.63\right)$, meaning that 4 group members obtain a reward through costly investment, 1 non-investor obtains a reward for free, and 1 non-investor gets no reward. The 4 subjects with a reward feel a small sense of relative deprivation by comparing to the 1 group member with a free reward, and the 1 subject without reward feels a moderate level of relative deprivation by being worse off than all group members, but only slightly so because he/she did not make the costly investment. The combination of the small and moderate relative deprivation of subjects under the observed scenario is about equal to the strong sense of 
relative deprivation of the single actor under our hypothetical scenario. Therefore, both scenarios predict a similar average level of relative deprivation, despite completely different investment behaviour. A different but related question is what drives the subjects to invest differently from what we predicted in the high mobility condition. We discuss possible answers to this question and directions for future research in Section 5.

Finally, recall that our general simulations predicted that decreasing investments would induce a second inverse U-shape between improving social mobility and relative deprivation, because it first shifts the actors from a position of equality (many actors earn a reward through investment) to a position of larger interpersonal differences (several actors who earn a reward with and without investment), and then to position of equality again (many actors earn a reward without investment). Although we did not explicitly test this prediction, the finding that subjects increase their investments even when we expected them to almost completely stop investing implies that such a second inverse Ushape would have been unlikely in the experiment.

\section{Conclusion and discussion}

Although it is known that more upward social mobility within a group can sometimes paradoxically lead to more frustration (relative deprivation) among its members, we have little understanding of the conditions under which this paradox occurs. We used an extended version of Boudon's game-theoretic model of relative deprivation (1982 [1977]) to derive these conditions. In the model, actors choose whether to make a costly investment for a better chance of obtaining one of a limited number of rewards. Through game-theoretic computer simulations on this model, we predicted how improving social mobility, through a micro-process of investments and comparisons of interpersonal differences, affect the macro-level relative deprivation. We conducted a laboratory experiment to examine a subset of the predictions empirically.

The main prediction was that the paradox occurs if the cost of investment is low, and social mobility moves from a low to medium level. Under these conditions, the improvement causes an increase in investments that shifts the actors from a position of equality (majority no reward) to a position of larger interpersonal differences (several actors with and without reward) which intensifies the level of relative deprivation. Further improving the proportion of rewards from a medium to a high level brings the actors back to a position of equality (majority obtains a reward), and hence reduces the level of relative deprivation. In total, the path between increasing social mobility and relative deprivation under low investment costs was thus predicted to be inversely U-shaped. Under a high cost of investment, we predicted relative deprivation to remain rather constant over increasing social mobility; high investment costs imply that the net differences (benefits of reward minus investment costs) between investors with a reward and non-investors without reward are small. Both predicted paths of relative deprivation were corroborated by the experimental test. What is more, our point predictions on the magnitude of relative deprivation within mobility settings also found support. Thus, not only did both research methods align in when relative deprivation increases, but also by how much it increases.

Interestingly, the predictions for the investment behaviour assumed to underlie these changes in relative deprivation received less support from the experiment. We did correctly predict that investments increase when social mobility moves from a low to medium level (the conditions that bring about the paradox) but could in general not correctly predict investments under a high level of social mobility. In particular, most subjects did not attempt to obtain a reward 'for free' by ceasing to invest when mobility improves from a medium to a high level and investment costs are high. Post-hoc analyses suggest that this mismatch with the simulation results that did predict such a decrease in investments may be explained by the subjects' beliefs on how many other subjects will invest. In the high mobility condition (with 5 rewards for 6 actors), subjects can only hope to obtain a reward without investment if at least one other actor would also not invest. Our experimental data show that $77 \%$ of the investors believed all other actors to invest, while only $30 \%$ of the non-investors believed all other actors to invest. The large majority of investors thus had no hope of receiving a reward without investment, while the large majority of non-investors did deem a reward without investment possible. This suggests that the primary reason why many subjects still invest in the high mobility condition is their belief that they will not be able to get a reward without investment, a belief our simulated actors did not have.

Still, we cannot strictly rule out that changes in other micro-behavioural assumptions would also improve our understanding of the subjects' behaviour. Of specific interest in this regard is our newly introduced preference for inequity aversion to the model. With this preference, actors not only care about increasing their own payoff, but also care about not being disadvantaged to others. In this paper, we simulated the population-level relationship between social mobility and relative deprivation under this behavioural rule. Although a population of actors with (heterogeneous and private) inequity aversion behaves differently than a population of payoff-maximizing actors, which is captured in our model, we did not explicitly analyse the influence of individual inequity aversion on individual investment behaviour and relative deprivation. To get a fuller understanding of how different micro-behavioural rules and sensitivities to inequity aversion affect investment behaviour, our model can also be used to simulate microlevel data, i.e., the probability for investment for a wide range of inequity aversion parameters. By applying statistical analysis to the micro-data, hypotheses can be derived on how the individual investment decisions depend on the individual inequity aversion as well as on the individual beliefs and macro-parameters (group size, mobility). These hypotheses can be tested by analysing our experimental data using subject-level, random-effects, models. Using these statistical models, it would be possible to estimate inequity aversion as a latent trait for each subject and assess its influence on relative deprivation. We believe this to be an insightful direction for future research on the model's micro-behavioural patterns.

Despite the unexpected finding that subjects do not strategically try to obtain rewards without investment, the majority of our simulation predictions were supported by the laboratory experiment. This corroboration from two research methods gives us more confidence in the results on the relationship between macro-level social mobility and macro-level frustration (relative deprivation). Our results suggest that the relationship may be paradoxical, as when the social mobility moves from a low to medium level and the costs of investment in a better position are low, but it does not need to be. When social mobility moves from a medium to a high level, and the costs of investment in a better position are low, we find a decrease in relative deprivation instead. When the costs of investment in a better position are high, relative deprivation can remain largely stable over increasing levels of social mobility.

The model is of course still a highly simplistic view of real-life situations of competition and relative deprivation. Several extensions are possible to further increase its application to real-life settings, and in particular to status competition through educational investments. These include the incorporation of relative gratification alongside relative deprivation (see Runciman, 1966), potential network effects within groups (see Manzo, 2011), repeated plays of the model (Ishida, 2012) and actor heterogeneity in the cost-to-benefit ratios of investing and the chances of status attainment (Yamaguchi, 1998). We have not seen, but are interested in, extensions that allow for more than two levels of investment and more than two types of status positions. Such modifications can be straightforwardly implemented in laboratory experiments.

In making the model less abstract and more realistic, we would eventually also be able to perform empirical tests outside of the laboratory. The data requirements to do so are small. Take the 
application to investments in education for high-status positions as an example again. National data on the number and type of job vacancies and the number and type of occupied positions are available at least on a year-to-year basis for most countries, providing information on the changing macro-level opening and allocation of positions. National data on educational investment costs could for many countries be derived, for example from (year-to-year) tuition fees and expected foregone wages. Survey data on individual behaviour and satisfaction on the labour market could be integrated with these national data to also incorporate the micro-level approach.

Eventually, such tests outside of the simulation and laboratory environment are necessary as each method has its specific limitations. It is difficult for formal models and simulations (and probably also undesirable) to incorporate all of the complexity involved in human decision making and the social environment in which the decision making takes place. Lab experiments allow the researcher to test the model in an environment almost as tightly controlled as the simulations, but with the additional complexity of real human behaviour. Still, the human subjects in experiments are typically not representative of the general population of interest. Moreover, in real-life situations the decision to invest in for example a higher level of education is rarely a simple yes or no, and often involves considerations that we abstracted away in the experiment. In addition, lab experiments often have more difficulty in finding effects of small size due to limitations on the number of subjects that can be included. Empirical data outside of the lab, for instance through surveys or archival material, could help to study the model with a representative sample, in a more natural context, and under a larger set of parameter values.

Yet, the advantages of combining modelling, simulation, and lab experimentation should not be overlooked. Through generating and analysing formal models, the micro-level mechanisms believed to underlie the macro-level phenomenon of interest are made explicit, and precise hypotheses to test these mechanisms can be derived. This aids in a causal understanding of the phenomenon, which can then be tested in the tight control of a laboratory experiment. We believe that this approach can be a useful complement to the more traditional approaches in stratification research that emphasize statistical associations and survey analysis. We hope that the present study on Boudon's model of social mobility and relative deprivation serves as a modest example of the insights that modelling, simulation, and experimentation can offer.

\section{Declaration of Competing Interest}

None.

\section{Acknowledgements}

The author would like to express his gratitude to Jeroen Weesie, who offered many important insights, comments, and ideas. The author also sincerely thanks Rense Corten and Vincent Buskens for commenting on earlier draft versions of the manuscript, and two anonymous reviewers for their useful suggestions. Financial support for the data collection by Utrecht University is acknowledged.

\section{Appendix A. Supplementary data}

Supplementary material related to this article can be found, in the online version, at doi:https://doi.org/10.1016/j.rssm.2019.100440.

\section{Appendix A}

Table A1

Within-Subject Correlations Between Prior Outcomes and Current Investment Decision.

\begin{tabular}{|c|c|c|c|}
\hline Prior outcome & $\begin{array}{l}\text { Time period } \\
\text { prior outcome }\end{array}$ & $\begin{array}{l}\text { Nr. of } \\
\text { observations }\end{array}$ & $\begin{array}{l}\text { Correlation with current } \\
\text { investment choice }(\mathrm{t})\end{array}$ \\
\hline \multirow[t]{5}{*}{ Type $B_{L} C_{L}$} & $\mathrm{t}-1$ & 720 & -.01 \\
\hline & $\mathrm{t}-2$ & 576 & $.12^{* *}$ \\
\hline & $\mathrm{t}-3$ & 432 & .03 \\
\hline & $\mathrm{t}-4$ & 288 & .08 \\
\hline & $\mathrm{t}-5$ & 144 & .13 \\
\hline \multirow[t]{5}{*}{ Type $B_{L} C_{H}$} & $\mathrm{t}-1$ & 720 & $.17^{* * *}$ \\
\hline & $\mathrm{t}-2$ & 576 & $.09^{*}$ \\
\hline & $\mathrm{t}-3$ & 432 & -.09 \\
\hline & $\mathrm{t}-4$ & 288 & $-.16^{* *}$ \\
\hline & $t-5$ & 144 & -.13 \\
\hline \multirow[t]{5}{*}{ Type $B_{H} C_{H}$} & $\mathrm{t}-1$ & 720 & .00 \\
\hline & $\mathrm{t}-2$ & 576 & $-.13^{* *}$ \\
\hline & $t-3$ & 432 & .01 \\
\hline & $\mathrm{t}-4$ & 288 & .02 \\
\hline & $t-5$ & 144 & -.08 \\
\hline \multirow{4}{*}{ Type $B_{H} C_{L}$} & $\mathrm{t}-2$ & 576 & $-.13^{* *}$ \\
\hline & $t-3$ & 432 & .08 \\
\hline & $\mathrm{t}-4$ & 288 & $.12^{*}$ \\
\hline & $\mathrm{t}-5$ & 144 & $.18^{*}$ \\
\hline \multirow[t]{5}{*}{ Total points } & $\mathrm{t}-1$ & 720 & $-.13^{* * *}$ \\
\hline & $\mathrm{t}-2$ & 576 & -.03 \\
\hline & $\mathrm{t}-3$ & 432 & $.16^{* * *}$ \\
\hline & $\mathrm{t}-4$ & 288 & $.17^{* *}$ \\
\hline & $\mathrm{t}-5$ & 144 & .06 \\
\hline
\end{tabular}

read $\mathrm{t}-1$ as one round prior.

$* \mathrm{p}<.05$.

$* * \mathrm{p}<.01$.

$* * * \mathrm{p}<.001$. 
Table A2

Population-Averaged Logit Regression for Probability of Investment and Population-Averaged Linear Regression for Relative Deprivation.

\begin{tabular}{lcc}
\hline & Prob. of Investment & Relative Deprivation \\
\hline low $Q, 1$ reward & $.49^{* * *}$ & $14.64^{* * *}$ \\
& $(.04)$ & $(1.45)$ \\
low $Q, 3$ rewards & $.88^{* * *}$ & $23.06^{* * *}$ \\
& $(.03)$ & $(1.46)$ \\
low $Q, 5$ rewards & $.93^{* * *}$ & $12.48^{* * *}$ \\
& $(.02)$ & $(1.45)$ \\
high Q, 1 reward & $.12^{* * *}$ & $7.70^{* * *}$ \\
& $(.03)$ & $(1.45)$ \\
high $Q, 3$ rewards & $.41^{* * *}$ & $10.10^{* * *}$ \\
& $(.04)$ & $(1.45)$ \\
high $Q, 5$ rewards & $.63^{* * *}$ & $11.27^{* * *}$ \\
& $(.04)$ & $(1.45)$ \\
$R^{2}$ & .35 & .10 \\
$N_{\text {obs }}$ & 864 & 864 \\
$N_{\text {subjects }}$ & 144 & 144
\end{tabular}

Coefficients are predictive margins, for the logit model reported in probabilities. Standard error in parentheses. The within-subject "working correlation matrix" is unstructured. We use no base level for the conditions, and therefore leave out a fixed intercept. $Q$ is the cost-to-benefit ratio of investing (investment costs). $\mathrm{R}^{2}$ logit model calculated based on Tjur (2009). $\mathrm{R}^{2}$ linear model calculated based on Hardin and Hilbe (2003). Controlled for session (not shown).

$* * * \mathrm{p}<.001$ (two-tailed tests).

Table A3

Selection of Pairwise Comparisons, Based on Models Under Table A2.

\begin{tabular}{|c|c|c|c|}
\hline Investment costs & Nr. of rewards & Prob. of Investment & Relative Deprivation \\
\hline low & $1 \rightarrow 3$ & $\begin{array}{l}.40^{* * *} \\
(.05)\end{array}$ & $\begin{array}{l}8.43^{* * *} \\
(2.05)\end{array}$ \\
\hline low & $3 \rightarrow 5$ & $\begin{array}{c}.05 \\
(.03)\end{array}$ & $\begin{array}{c}-10.59^{* * *} \\
(1.99)\end{array}$ \\
\hline high & $1 \rightarrow 3$ & $\begin{array}{l}.29^{* * *} \\
(.05)\end{array}$ & $\begin{array}{c}2.41 \\
(2.02)\end{array}$ \\
\hline high & $3 \rightarrow 5$ & $\begin{array}{l}.22^{* * * *} \\
(.05)\end{array}$ & $\begin{array}{c}1.16 \\
(2.02)\end{array}$ \\
\hline
\end{tabular}

Standard error in parentheses. We take multiple comparisons into account through Bonferroni correction (per outcome 8 comparisons). $* * * \mathrm{p}<.001$ (two-tailed tests).

Table A4

Equivalence Test for Directional Hypotheses, Based on Models Under Table A2.

\begin{tabular}{lllll}
\hline Outcome & $\begin{array}{l}\text { Nr. of } \\
\text { rewards }\end{array}$ & $\begin{array}{l}\text { Investment } \\
\text { costs }\end{array}$ & $\begin{array}{l}\text { Equivalence } \\
\text { range }\end{array}$ & $\begin{array}{l}\text { Observed } 90 \% \\
\text { CI }\end{array}$ \\
\hline$p_{C H}$ & $3 \rightarrow 5$ & low & {$[-.08, .08]$} & {$[-6.00,6.00]$} \\
$R D$ & $1 \rightarrow 3$ & high & {$[-6.00,6.00]$} & {$[-.91,5.72]^{*}$} \\
$R D$ & $3 \rightarrow 5$ & high & {$[-2.16,4.49]^{*}$} & \\
\hline
\end{tabular}

Equivalence range obtained by adding and subtracting $7.5 \%$ of the maximum possible value of the outcome (.08 for $p_{C H}$ and 6.00 for $R D$ ) to and from zero. To prevent inadmissible ranges, both the lower bound of the equivalence range and $\mathrm{CI}$ are cut off at the minimum possible value. $p_{C H}=$ probability of investment. $R D=$ relative deprivation.

* Observed $90 \%$ CI falls within equivalence range; significant equivalence. 
Table A5

Equivalence Tests for Point Hypotheses, Based on Models Under Table A2.

\begin{tabular}{|c|c|c|c|c|}
\hline Outcome & $\begin{array}{l}\text { Nr. of } \\
\text { rewards }\end{array}$ & $\begin{array}{l}\text { Investment } \\
\text { costs }\end{array}$ & $\begin{array}{l}\text { Equivalence } \\
\text { range }\end{array}$ & $\begin{array}{l}\text { Observed } 90 \% \\
\text { CI }\end{array}$ \\
\hline$p_{C H}$ & 1 & low & {$[.45, .60]$} & {$[.42, .56]$} \\
\hline$p_{C H}$ & 3 & low & {$[.76, .91]$} & {$[.84, .93]$} \\
\hline$p_{C H}$ & 5 & low & {$[.78, .93]$} & {$[.89, .96]$} \\
\hline$p_{C H}$ & 1 & high & {$[.15, .30]$} & {$[.08, .16]$} \\
\hline$p_{C H}$ & 3 & high & {$[.37, .52]$} & {$[.35, .48]$} \\
\hline$p_{C H}$ & 5 & high & {$[.00, .13]$} & {$[.57, .69]$} \\
\hline$R D$ & 1 & low & {$[7.66,19.66]$} & {$[12.25,17.03]^{*}$} \\
\hline$R D$ & 3 & low & {$[15.40,27.40]$} & {$[20.67,25.46]^{*}$} \\
\hline$R D$ & 5 & low & {$[5.73,17.73]$} & {$[10.10,14.86]^{*}$} \\
\hline$R D$ & 1 & high & {$[0.20,12.20]$} & {$[5.31,10.09]^{*}$} \\
\hline$R D$ & 3 & high & {$[3.03,15.03]$} & {$[7.71,12.50]^{*}$} \\
\hline$R D$ & 5 & high & {$[4.53,16.53]$} & {$[8.88,13.66]^{*}$} \\
\hline
\end{tabular}

Note: Equivalence range obtained by adding and subtracting $7.5 \%$ of the maximum possible value of the outcome (.08 for $p_{C H}$ and 6.00 for $R D$ ) to and from the predicted point. To prevent inadmissible ranges, both the lower bounds of the equivalence range and $\mathrm{CI}$ are cut off at the minimum possible value. $p_{C H}=$ probability of investment. $R D=$ relative deprivation.

* Observed 90 \% CI falls within equivalence range; significant equivalence.

\section{References}

Arrow, K. J. (1973). Higher education as a filter. Journal of Public Economics, 2(3), 193-216.

Berger, J., \& Diekmann, A. (2015). The logic of relative frustration: Boudon's competition model and experimental evidence. European Sociological Review, 31(6), 725-737.

Boudon, R. (1974). Education, opportunity, and social inequality. New York: Riley.

Boudon, R. (1979). Generating models as a research strategy. In R. K. Merton, J. S. Coleman, \& P. H. Rossi (Eds.). Qualitative and quantitative social research (pp. 51-64). New York: The Free Press.

Boudon, R. (1982 [1977). The logic of relative frustration. The unintended consequences of social action. London: The Macmillan Press Ltd105-126.

Brinton, C. (1938). A summary of the work of revolutions. The anatomy of revolution. New York: Vintage Books272-302.

de Tocqueville, A. (1952 [1856). That the reign of Louis XVI. was the most prosperous era of the old monarchy, and how that prosperity really hastened the revolution. The old regime and the revolution. Paris: Editions Gallimard206-217.

Easterlin, R. A. (2001). Income and happiness: Towards a unified theory. The Economic Journal, 111(473), 465-484.

Fehr, E., \& Schmidt, K. M. (1999). A theory of fairness, competition, and cooperation. The Quarterly Journal of Economics, 114(3), 817-868.

Fischbacher, U. (2007). z-Tree: Zurich toolbox for ready-made economic experiments. Experimental Economics, 10(2), 171-178.

Greiner, B. (2015). Subject pool recruitment procedures: Organizing experiments with ORSEE. Journal of the Economic Science Association, 1(1), 114-125.

Hardin, J. W., \& Hilbe, J. M. (2003). Generalized estimating equations. Generalized estimating equations. Boca Raton, FL: Chapman \& Hall/CRC Press55-134.

Harsanyi, J. C. (1968). Games with incomplete information played by Bayesian players. Management Science, 14, 468-502.

Ishida, A. (2012). An Evolutionary Game Analysis of the Boudon-Kosaka Model of Relative Deprivation. Retrieved from Kwansei Gakuin University Repository: http:// hdl.handle.net/10236/9012.

Johansson-Stenman, O., Carlsson, F., \& Daruvala, D. (2002). Measuring future grandparents' preferences for equality and relative standing. The Economic Journal, $112(479), 362-383$.

Kosaka, K. (1986). A model of relative deprivation. The Journal of Mathematical Sociology, $12,35-48$.

Manzo, G. (2009). Boudon's model of relative deprivation revisited. In M. Cherkaoui, \& P. Hamilton (Eds.). Raymond Boudon-A life in sociology. Essays in honour of Raymond Boudon (pp. 91-121). Oxford: Bardwell.

Manzo, G. (2011). Relative deprivation in silico: Agent-based models and causality in analytical sociology. In P. Demeulenaere (Ed.). Analytical sociology and social mechanisms (pp. 266-308). Cambridge: Cambridge University Press.

McKelvey, R. D., \& Palfrey, T. R. (1995). Quantal response equilibria for normal form games. Games and Economic Behavior, 10(1), 6-38.

Merton, R. K., \& Rossi, A. S. (1968). Contributions to the theory of reference group behavior. In R. K. Merton (Ed.). Social theory and social structure (pp. 215-248). New
York: The Free Press.

Oberschall, A. (1970). Group violence: Some hypotheses and empirical uniformities. Law \& Society Review, 5(1), 61-92.

Otten, K. (2018). Laboratory Experiment on a New Version of Boudon's Game-Theoretic Model of Relative Deprivation. Available from DANS: https://doi.org/10.17026/ dans-xjw-449u.

Pettigrew, T. F. (2015). Samuel Stouffer and relative deprivation. Social Psychology Quarterly, 78(1), 7-24.

Raub, W. (1984). Interdependenzen, individuelles Verhalten und kollektive Effekte: Wettbewerbsstrukturen, Relative Deprivation und suboptimale Ressourcenallokation. Rationale Akteure, institutionelle Regelungen und Interdependenzen. Untersuchungen zu einer erklärenden Soziologie auf strukturell-individualistischer Grundlage. Frankfurt am Main: Peter Lang195-295.

Reyniers, D. J. (1998). Deprivation in heterogeneous organizations. The Journal of Mathematical Sociology, 23(1), 59-76.

Rosenbaum, J. E. (1979). Organizational career mobility: Promotion chances in a corporation during periods of growth and contraction. The American Journal of Sociology, 85(1), 21-48.

Runciman, W. G. (1966). Relative deprivation and the concept of reference. Relative deprivation and social justice: A study of attitudes to social inequality in twentieth-century England. London: Routledge and Kegan Paul9-35.

Smith, H. J., Pettigrew, T. F., Pippin, G. M., \& Bialosiewicz, S. (2012). Relative deprivation: A theoretical and meta-analytic review. Personality and Social Psychology Review, 16(3), 203-232.

Solon, G., Whatley, W., \& Stevens, A. H. (1997). Wage changes and intrafirm job mobility over the business cycle: Two case studies. ILR Review, 50(3), 402-415.

Stevenson, B., \& Wolfers, J. (2013). Subjective well-being and income: Is there any evidence of satiation? The American Economic Review, 103(3), 598-604.

Stiglitz, J. E. (1975). The theory of "screening", education, and the distribution of income. The American Economic Review, 65(3), 283-300.

Stouffer, S. A., Suchman, E. A., de Vinney, L. C., Star, S. A., \& Williams, R. M., Jr. (1965 [1949). Adjustments during army life. The American soldier. Manhattan (Kansas): Military Affairs, Aerospace Historian Publishing125-181.

Thurow, L. C. (1976). Generating inequality. London: Macmillan129-154.

Tjur, T. (2009). Coefficients of determination in logistic regression models-A new proposal: The coefficient of discrimination. The American Statistician, 63(4), 366-372.

Walker, E., \& Nowacki, A. S. (2011). Understanding equivalence and noninferiority testing. Journal of General Internal Medicine, 26(2), 192-196.

Walker, I., \& Smith, H. (2002). Relative deprivation: Specification, development, and in tegration. Cambridge: Cambridge University Press.

Yamaguchi, K. (1998). Rational-choice theories of anticipatory socialization and anticipatory non-socialization. Rationality and Society, 10(2), 163-199.

Yitzhaki, S. (1979). Relative deprivation and the Gini coefficient. The Quarterly Journal of Economics, 3, 321-324.

Zeger, S. L., Liang, K.-Y., \& Albert, P. S. (1988). Models for longitudinal data: A generalized estimating equation approach. Biometrics, 44, 1049-1060. 